\title{
Características composicionales y calidad higiénica de leche de cabra de sistemas de producción de la provincia de García Rovira, Santander (Colombia)
}

Characteristics of compositional quality and hygienic quality of goat's milk from production systems in the García Rovira province, Santander (Colombia)

Caraterísticas composicionais e qualidade higiênica do leite de cabra em sistemas de produção da Provincia de Garcia Rovira, Santander

(Colômbia)

\author{
Felipe Torres-Ruda ${ }_{1,2}$ \\ Mónica Torres-Cruz ${ }_{1,2}$ \\ Carlos Ariel Joya-Cárdenas \\ Frank Castellanos-Duarte ${ }_{1}{ }_{1}$
}

Recibido: 15 de enero de 2021

Aprobado: 28 de marzo de 2021

Publicado: 3 de mayo de 2021

Cómo citar este artículo:

Torres-Ruda F, Torres-Cruz M, Joya-Cárdenas CA, Castellanos-Duarte F. Características composicionales y calidad higiénica de leche de cabra de sistemas de producción de la provincia de García Rovira, Santander (Colombia). Spei Domus. 2021;17(1): 1-14. doi: https://doi.org/10.16925/2382-4247.2021.01.06

Artículo de investigación. https://doi.org/10.16925/2382-4247.2021.01.06

* $\quad$ Autor de correspondencia: Frank Castellanos Duarte.

Correo electrónico: facastellanos10@misena.edu.co

Centro Agroempresarial y Turístico de los Andes, Málaga, Santander - SENNOVA. Grupo de investigación Frailejones. Semillero de investigación en Caprinos y Ovinos- SIPCO.

Departamento de Producción Animal, Facultad de Medicina Veterinaria y de Zootecnia. Universidad Nacional de Colombia, Sede Bogotá. Grupo de investigación: Gestión tecnológica e innovación en sistemas pecuarios. SIGETEC. 
2 Características composicionales y calidad higiénica de leche de cabra de sistemas de producción de la provincia de García Rovira, Santander (Colombia)

\section{Resumen}

Introducción: la leche caprina es reconocida como un alimento con compuestos de alto valor biológico dada su composición y sus características intrínsecas, por lo que alcanza un alto reconocimiento y valor comercial, sin embargo, los sistemas de producción de la región se han desarrollado de manera aislada, sin acompañamiento técnico, ausencia de registros y baja inclusión tecnológica, desconociendo los valores mínimos de calidad a través de normativas que regulen su producción y comercialización. Por ello, el objetivo del presente trabajo fue reconocer características de composición y calidad higiénica de la leche de cabra.

Metodología: análisis los parámetros fisicoquímicos densidad $\left(\mathrm{g} / \mathrm{cm}^{3}\right)$, grasa (\%), sólidos no grasos (SNG) (\%), proteína (\%) y punto crioscópico $\left({ }^{\circ} \mathrm{C}\right)$ utilizando el analizador de leche ultrasónico Milkana Kam $98-2 \mathrm{~A}$ marca Ekomilk®. en unidades productivas-UP ubicadas en la provincia de García Rovira en el departamento de Santander.

Resultados: los valores promedio para las variables fisicoquímicas fueron: $4,44 \pm 1,66 \%$, en grasa, $3,31 \pm 0,24 \%$ en proteína, $8,77 \pm 0,65 \%$ en sólidos no grasos $-S N G, 1,031 \pm 0,03 \mathrm{~g} / \mathrm{cm}^{3}$ para densidad $\mathrm{y},-0,561 \pm 0,06{ }^{\circ} \mathrm{C}$ para el punto crioscópico; la prueba azul de reductasa -TRAM mostró que el 73 \% de la leche se califica como grado II y III, lo cual es aceptado para el consumo humano, además, el $80 \%$ de los animales de las unidades productivas -UP evaluados fueron negativos a la prueba de California Mastitis Test -CMT.

Conclusión: estos datos contribuyen con información referencial fundamental para apoyar la estructuración de aspectos de carácter normativo para la comercialización de leche de cabra en el país.

Palabras clave: composición láctea, industria lechera, normatividad, ordeño, zootecnia.

\section{Abstract}

Introduction: Goat milk is recognized as a food with compounds of high biological value given its composition and intrinsic characteristics, reaching high recognition and commercial value. However, the production systems of the region have been developed in isolation, without technical support, absence of records and low technological inclusion, ignoring limited quality values through regulatory standards that regulate their production and extension. Therefore, the objective of this work was to recognize characteristics of composition and hygienic quality of goat's milk in farms located in the García Rovira province in the department of Santander. Methods: Analysis of the physicochemical parameters density (g / cm3), fat (\%), non-fat solids (MSNF) (\%), protein $(\%)$ and cryoscopic point $\left({ }^{\circ} \mathrm{C}\right)$ using the brand Milkana Kam 98-2A ultrasonic milk analyzer Ekomilk ${ }^{\circledR}$. Results: The means values for the physicochemical variables were: $4.44 \pm 1.66 \%$, in fat, $3.31 \pm 0.24 \%$ in protein, $8.77 \pm 0.65 \%$ in SNG, $1.031 \pm 0.03 \mathrm{~g} / \mathrm{cm} 3$ for density and, $-0.561 \pm 0.06^{\circ} \mathrm{C}$ for the cryoscopic point; the TRAM test also showed that $73 \%$ of the milk is classified as grade II and III, which is accepted for human consumption, in addition $80 \%$ of the animals of the UP evaluated were negative to the CMT test.

Conclusions: These specific data with fundamental referential information that can help in structuring aspects of a regulatory nature for the consumption of goat's milk in the country.

Keywords: Dairy industry, animal husbandry, milk collection, quality goat milk, normativity.

\section{Resumo}

Introdução: O leite caprino é reconhecido como um alimento com compostos de alto valor biológico segundo a sua composição e caraterísticas intrínsecas, por consequência um alto valor comercial, porém, os sistemas de produção foram desenvolvidos de forma isolada sem acompanhamento técnico, sem registro e baixa inclusão tecnológica, desconhecendo os valores mínimos de qualidade segundo as normativas que validam sua 
produção e comercialização. Por isto, o objetivo do presente estudo foi avaliar caraterísticas de composição e qualidade higiênica do leite de cabra.

Metodologia: Análise de parâmetros químicos de densidade $(\mathrm{g} / \mathrm{cm} 3)$, gordura $(\%)$, sólidos não gordurosos (SNG), proteína (\%) e índice crioscópico $\left({ }^{\circ} \mathrm{C}\right)$ utilizando o analisador do leite ultrassónico Milkana Kam 98-2A marca Ekomilk $\circledast$ nas unidades produtivas (UP) localizadas na Provincia de Garcia Rovira no departamento Santander.

Resultados: Os valores meios das variáveis físico-químicas foram: 4,44 $\pm 1,66 \%$ na gordura, 3,31 $\pm 0,24 \%$ na proteína, $8,77 \pm 0,65 \%$ no SNG, $1,031 \pm 0,03 \mathrm{~g} / \mathrm{cm} 3$ para densidade e, $-0,561 \pm 0,06{ }^{\circ} \mathrm{C}$ para o índice crioscópico; 0 teste de redutase TRAM apresentou que o $73 \%$ do leite tem qualidade tipo I e III, portanto é considerada apta para o consumo humano, além disso o $80 \%$ dos animais das UP foram negativos para a prova de California Mastites Test-CMT.

Conclusão: Estes dados contribuem na estruturação de informação referencial fundamental para o apoio de caráter normativo para a comercialização do leite de cabra no país.

\section{Introducción}

Los sistemas de producción de cabras tienen importancia en el mundo debido a su capacidad de adaptación, versatilidad y por la función que cumplen sus productos en la población (leche y carne) [1], [2]. El inventario mundial de cabras presenta que más de 1.034 millones de cabezas producen cerca de 18.656 millones de toneladas métricas de leche; un 53,8 \% de estas se encuentran en Asia, seguido de África (24,9\%), Europa (17,5\%) y las Américas (4,8 \%) [3]. Así mismo, la producción de leche de cabra en el mundo representa el 1,9\%, del total de leche producida por las diferentes especies, siendo India el mayor productor, con más del 21 \% de la producción mundial, cerca de 4 millones de toneladas de leche [3].

En Colombia, la población caprina se calcula en 1.034.615 animales, ubicados en su gran mayoría en los departamentos de la Guajira (79,4 \%), Boyacá (3,9\%), Cesar (3,5\%), Magdalena (3,3\%), Santander (2,9\%) y el resto en otras regiones (7\%) [4]. Respecto a la producción de leche, esta es marginal, dadas las cifras escasas, la centralización de la producción y la baja trazabilidad dentro de los sistemas; sin embargo, el consumo se ha proyectado al mediano plazo en 1,5 kg de leche per cápita [5].

En departamentos con tradición en sistemas de producción caprina como Santander, la cabra se encuentra arraigada a la región del Cañón del Chicamocha, la cual, abarca cerca del $7 \%$ de la superficie del departamento, y tiene características agroecológicas propias que le han permitido a esta especie sobrevivir y reproducirse bajo estas condiciones; dentro de la zona en mención, la leche de cabra presenta una alta demanda asociada a condiciones culturales de sus pobladores [6]. 
4 Características composicionales y calidad higiénica de leche de cabra de sistemas de producción de la provincia de García Rovira, Santander (Colombia)

Los sistemas de producción en esta región, basados en la intervención de carácter tradicional y cultural, se han desarrollado de manera aislada, sin acompañamiento técnico permanente, ausencia de registros, baja inclusión tecnológica, escasa planeación y organización [7], con mucho uso del conocimiento empírico por parte de los productores, pero con deficiencias en los procesos de adquisición y aplicación del conocimiento científico o tecnológico, han crecido lenta y dispersamente [7]. Sin embargo, pese a los limitantes, la producción merece importancia, dada la capacidad de adaptación de las cabras a condiciones adversas, por su estructura anatómica y morfológica, permitiéndole la utilización eficiente de alimento de baja calidad [8] y mantener su productividad, además, los productos obtenidos de las mismas, son una fuente primaria de alimentos, que contribuyen a la seguridad alimentaria de la comunidad y a combatir el cambio climático y la desertificación [9].

La leche caprina es reconocida como un alimento con compuestos de alto valor biológico como proteínas, ácidos grasos, minerales, vitaminas y factores alergénicos, que puede sustituir la leche de vaca con algunas ventajas [10]. En este sentido, la leche y sus productos derivados presentan beneficios en cuanto al mantenimiento de la salud, las funciones fisiológicas en la nutrición de los niños y los adultos mayores [11]; además, ha empezado a ser reconocida por sus características intrínsecas, alcanzando un alto valor comercial [12]. Igualmente, la composición puede variar por factores como: genética, edad, tipo de alimentación, tipo de nacimiento, duración de la lactancia, periodo ambiental o estacional, tipo de ordeño, entre otros, los cuales interfieren en mayor o menor grado en la calidad de la misma [13].

Cabe señalar que se hace necesario empezar a indagar y aportar datos referenciales que permitan conocer y comparar los criterios mínimos sobre los cuales se produce y comercializa la leche para consumo humano, tal y como se documenta en el Decreto 616 de 2006 del Ministerio de Agricultura y Desarrollo Rural (MADR), el cual estandarizó para la ganadería bovina los parámetros fisicoquímicos y microbiológicos propios de la leche. Por ello, el objetivo de este estudio fue reconocer algunas características de composición y calidad higiénicas de la leche de cabra en fincas ubicadas en la provincia de García Rovira en el departamento de Santander con el fin de contribuir a la estandarización de parámetros de calidad para la comercialización de leche de cabra en la región. 


\section{Materiales y métodos}

\section{Ubicación geográfica y selección de predios}

El estudio fue llevado a cabo en quince unidades de producción (UP) ubicadas en la provincia de García Rovira en el departamento de Santander, zona reconocida por su ubicación sobre el cañón del Chicamocha y clasificada como bosque seco pre montano espinoso [14], con fuertes vientos cálidos y pendientes pronunciadas; de acuerdo a los reportes del Instituto de Hidrología, Meteorología y Estudios Ambientales (IDEAM), esta zona geográfica presenta una temperatura promedio de $19 \pm 3{ }^{\circ} \mathrm{C}$, precipitación

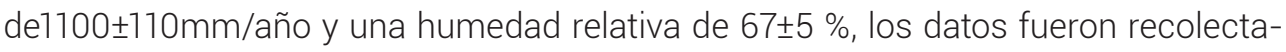
dos durante los meses de junio (periodo lluvioso) y noviembre (periodo seco) de 2018. Las UP se caracterizaban por desarrollar un manejo en condiciones de semipastoreo, con bajo nivel de inclusión tecnológica (ordeño manual), un ordeño al día durante las horas de la mañana, con un promedio de cabras lactando de 15 \pm 13 animales; estos fueron los criterios de inclusión para seleccionar las UP.

\section{Toma de muestras}

Para determinar la composición físico-química de la leche, se tomaron 30 muestras de los lotes provenientes de las UP, dedicadas a la comercialización y transformación de leche en la zona. La recolección de las muestras para su evaluación composicional se dio de acuerdo con el protocolo establecido por Laboratorio de Biotecnología Reproductiva y Sanidad Animal del Centro Agroempresarial y Turístico de los Andes; el volumen de cada una fue $30 \pm 5 \mathrm{ml}$, obtenido directamente del tanque de almacenamiento de cada UP. Las muestras fueron almacenadas y conservadas a $4 \pm 2{ }^{\circ} \mathrm{C}$ hasta el momento del análisis. Para el diagnóstico de mastitis en las UP, se evaluaron 145 hembras para el periodo lluvioso, y 123 para el periodo seco, mediante el uso de la prueba California Mastitis Test (CMT). Se tomó muestra de cada medio, para un total de 536 muestras evaluadas.

\section{Pruebas diagnósticas}

Los parámetros fisicoquímicos densidad $\left(\mathrm{g} / \mathrm{cm}^{3}\right)$, grasa (\%), sólidos no grasos (SNG) (\%), proteína (\%) y punto crioscópico $\left({ }^{\circ} \mathrm{C}\right)$ se determinaron con el analizador de leche ultrasónico Milkana Kam 98-2A marca Ekomilk®. La medición de carga microbiana se realizó bajo el protocolo de la prueba azul de reductasa o tiempo de reducción de azul de metileno (TRAM), método estandarizado por García et al. [15], con una 
6 Características composicionales y calidad higiénica de leche de cabra de sistemas de producción de la provincia de García Rovira, Santander (Colombia)

solución de azul de metileno de 50 mg/ml sugerida por Zambrano et al. [16] y se ajustó un método de clasificación de tres niveles: I, II, III, para determinar la calidad higiénica de la leche a partir de lo sugerido por Morales-Vanegas [17].

La detección de mastitis clínica se realizó mediante un examen físico de la glándula mamaria y se analizaron los primeros chorros de leche en un recipiente de fondo oscuro de acuerdo al procedimiento del CMT [18], el cual se clasifica en negativo, mastitis subclínica grado 1, 2 y 3 y positivo o clínico.

\section{Diseño estadístico}

Se realizó estadística descriptiva para determinar los valores de referencia de las variables de calidad físico-química: densidad, grasa, SNG, proteína y punto crioscópico de la leche evaluada; los valores para cada una de las variables se presentan como la media \pm la desviación estándar (DS). Mediante tablas de frecuencia se determinó la prevalencia de mastitis subclínica grado 1, 2 y 3 y clínica, en cada uno de los periodos evaluados, y la clasificación grado I, II, III para la prueba TRAM. Los datos fueron organizados y analizados mediante el software Microsoft Excel versión 2016®.

\section{Resultados y discusión}

Los valores de calidad físico-química evaluados se presentan en la tabla 1. Se observó que el porcentaje de grasa hallado fue en promedio de 4,4 4 ,6 \%, con un coeficiente de variación del $37 \%$, lo que indica heterogeneidad de los datos; los valores encontrados para la variable se encuentran acorde con los que se reportan en la literatura $4,8 \pm 0,17$ [19]. La leche de cabra se caracteriza por tener propiedades importantes en cuanto al contenido de grasa, su composición de ácidos grasos (AG) de cadena corta presenta mayores contenidos de C 6:0 y C 9:0 y aporta el sabor característico del producto [20], igualmente, esta leche posee una mayor digestibilidad, asociado a un menor tamaño de los glóbulos grasos comparado con la leche de vaca [21]; algunas características negativas de la leche son el bajo contenido de AG de cadena larga y los efectos negativos generados por la presencia de algunos AG de cadena corta que generan predisposición a enfermedades coronarias [22]

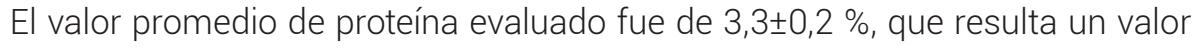
inferior al compararlo con lo reportado por Bedoya et al. [20] de 3,4\% y mayor que lo señalado por Ocampo et al. [23] de 3,01 \%, lo anterior deja en evidencia que la proteína evaluada está dentro del rango para la leche de cabra. Es necesario reconocer que el contenido de proteína influye en la presencia de algunos aminoácidos, los cuales son 
similares en la leche de cabra y de vaca; si bien, algunos están presentes solo en la leche caprina, como es el caso de la angiotensina, la cual brinda efectos hipotensores, otras proteínas presentes median la inflación, la progresión de tumores e incluso, contribuyen a la protección inmunológica de las crías [9]. Otro aspecto a destacar en la estructura de las proteínas de la leche de cabra es la menor cantidad de alergénicos presentes como las lactoalbuminas y las caseínas [9].

Con respecto a los SNG el valor promedio fue de 8,7 \pm 0,6 \%, dichos valores están por encima de lo descrito por Ocampo et al. [23] 7,99 \pm 0,30 \% y a los valores analizados por Herrera-Campos et al. [24] iguales a 8,12 \%, 8,06 y 8,00 \%; viéndose afectada esta variable por el tipo de alimentación a la cual se someten los animales.

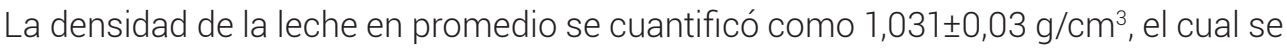
encuentra de los rangos reportados por Chacón [21], quien presenta valores entre 1,030 y 1,034 g/ $\mathrm{cm}^{3}$, enfatizando que los valores permitidos estén por encima de $1,028 \mathrm{~g} / \mathrm{cm}^{3}$; por el contrario a los planteamientos presentados por Kljajevic et al. [25], quienes refieren que durante las diferentes etapas de lactancia el valor promedio de densidad se mantiene constante con valores cercanos a 1,027 $\pm 0,001 \mathrm{~g} / \mathrm{cm}^{3}$.

El valor promedio para el punto de crioscopia fue de $-0,561 \pm 0,06{ }^{\circ} \mathrm{C}$, dicho valor está por debajo de lo mencionado por Kljajevic et al. [25], quienes evaluaron esta variable en leche proveniente de cabras Saanen se encontró valores entre -0,46 y -0,49 ${ }^{\circ} \mathrm{C}$ a través de las etapas de lactancia; ahora bien, de acuerdo con la reglamentación para la comercialización de leche bovina en el país, el presente estudio está por debajo del valor referencial en leches aptas para consumo humano $-0,53$ a $-0,51^{\circ} \mathrm{C}$.

Tabla 1. Variables de composición de la leche de cabra de UP de la región de García Rovira- Santander, Colombia

\begin{tabular}{lccccc}
\hline \multicolumn{1}{c}{ Variable } & Unidad & $\mathbf{n}$ & Media & DS & CV (\%) \\
\hline Grasa & $\%$ & 30 & 4,448 & 1,64 & 36,92 \\
\hline Proteína & $\%$ & 30 & 3,305 & 0,24 & 7,14 \\
\hline SNG & $\%$ & 30 & 8,771 & 0,65 & 7,42 \\
\hline Densidad & $\mathrm{g} / \mathrm{cm}^{3}$ & 30 & 1,0312 & 0,03 & 2,48 \\
\hline Punto crioscópico & ${ }^{\circ} \mathrm{C}$ & 30 & $-0,561$ & 0,06 & 10,70 \\
\hline
\end{tabular}

DS: Desviación Estándar, CV: Coeficiente de variación, SNG: Sólidos No Grasos

Fuente: elaboración propia.

Dado que se pretende establecer un referente de composición de la leche de cabra que permita conocer el rango de expresión de algunas variables, en la tabla 2 se 
8 Características composicionales y calidad higiénica de leche de cabra de sistemas de producción de la provincia de García Rovira, Santander (Colombia)

presentan resultados que han considerado algunos factores genéticos (tipos raciales), alimenticios y de ubicación, en los cuales se observa que animales nativos presentan en general, mayor porcentaje de proteína y SNG, posiblemente vinculado a los sistemas de alimentación y a características propias de las condiciones de manejo y/o del medio, tal y como se evidencia las UP donde se llevó a cabo la presente investigación, donde es frecuente encontrar una amplia variabilidad de composición botánica en la dieta, con especies que presentan un alto contenido de proteína. Asimismo, con respecto a la grasa, se observa que los animales de razas nativas presentan menores valores en comparación con las mestizas [26], [27].

Tabla 2. Variables referenciales de parámetros de calidad en leche de cabra

\begin{tabular}{lcccccc}
\hline Variable & & $\begin{array}{c}\text { Sanz et al., } \\
\mathbf{2 0 0 9}\end{array}$ & $\begin{array}{c}\text { Kljajevic } \\
\text { et al., } \mathbf{2 0 1 7}\end{array}$ & $\begin{array}{c}\text { Milewski } \\
\text { et al., } \mathbf{2 0 1 8}\end{array}$ & $\begin{array}{c}\text { Elmaz et al., } \\
\mathbf{2 0 1 8}\end{array}$ & $\begin{array}{c}\text { Sabah } \\
\text { et al., 2018 }\end{array}$ \\
\hline Tipo racial & Mestizos & Granadina & Saanen & Alpinas & Nativas & Nativas \\
\hline Tipo alimentación & Forraje & Mixto & Mixto & Mixto & ND & ND \\
\hline Ubicación & Colombia & España & India & Polonia & Turquía & Sudán \\
\hline Grasa \% & 4,45 & 5,23 & 3,41 & 4,23 & 1,4 & 3,9 \\
\hline Proteína \% & 3,31 & 3,48 & 2,79 & 3,39 & 4,2 & 4,81 \\
\hline SNG \% & 8,77 & 8,34 & 7,61 & ND & 10,4 & 10,7 \\
\hline Densidad g/cm ${ }^{3}$ & 1,03 & ND & 1,027 & ND & ND & ND \\
\hline
\end{tabular}

SNG: sólidos no grasos.

ND: dato no disponible a la fecha de la consulta.

Fuente: elaboración propia.

El estudio presentado por Kljajevic et al. [25] evaluó la incidencia del momento de la lactancia y encontraron que, al final de esta, hay mayor concentración de proteína, grasa y SNG, debido a que durante esta etapa se disminuye el volumen de producción y se eleva la concentración de nutrientes; para el caso de esta investigación, el 42 \% de los animales se encontraban en lactancia temprana, el $22 \%$ en lactancia media, el $5 \%$ en lactancia tardía y el 31 \% no presentaba información para determinar la misma, siendo relevante este factor para futuras investigaciones.

En alusión al tipo racial, Sanz et al. [28] reportaron mayores valores de grasa y proteína 5,23 y 3,48 \%, respectivamente, en comparación con el presente estudio, y SNG más bajos 8,34\%, siendo quizá el factor principal, el tipo de animales y sus razas especializadas, las cuales tienen un mayor volumen de producción y mayor duración de la lactancia. Por su parte, los valores promedio de grasa y proteína encontrados en cabras alpinas por Milewski et al. [29] son semejantes a los evaluados en este 
estudio; cabe resaltar que la distribución racial en las UP fue de $49 \%$ animales tipo mestizo, 29\% Alpinas Francesas, 11\% Santandereanas y el 11\% restante fue Saanen, Toggenburg, Alpina Americana y La Mancha. Es de anotar que, si bien los tipos de animales especializados han contribuido en el incremento de los volúmenes de producción, la duración de la lactancia en los diferentes tipos raciales y las condiciones de manejo de estas, varían de acuerdo con los sistemas productivos del país.

Los resultados obtenidos en referencia a la prueba TRAM indican que el 26,7\% de las muestras analizadas presentaron un tiempo de reacción corto ( $<4$ horas), lo que la clasifica como grado I, la cual se puede asociar con una alta presencia de microorganismos en la leche que afectan su calidad microbiológica; el 30 \% de las muestras obtuvo un tiempo de reacción entre 4 a 6 horas, lo que la cataloga como leche grado II, el 43,3 \% presentó un tiempo de más de 6 horas para su reacción lo que la clasifica como leche grado III. Teniendo en cuenta lo anterior, leches grado I pueden presentar contenidos mayores a 700.000 UFC/ml. Por otro lado, se menciona que a partir de 5 horas de reacción a la prueba, el número de bacterias estimado puede ser de 100.000 a 200.000 bacterias por $\mathrm{ml}$ [15], lo que permite clasificar la leche en grado II o III, aumentando su calidad higiénica ver tabla 3.

Tabla 3. Resultados prueba de reductasa TRAM, clasificados en tres niveles de calidad según las horas de reacción

\begin{tabular}{ccccc}
\hline TRAM $^{*}$ & Horas & $\mathbf{n}$ & $\mathbf{\%}$ & UFC/ml \\
\hline Grado I & $>4$ & 8 & 26,7 & $>700.000$ \\
\hline Grado II & $4-6$ & 9 & 30,0 & $200.000-700.000$ \\
\hline Grado III & $>6$ & 13 & 43,3 & $<200.000$ \\
\hline & & $\mathbf{3 0}$ & $\mathbf{1 0 0}$ & \\
\hline
\end{tabular}

UFC: unidades formadoras de colonia, TRAM: prueba de azul de reductasa.

Fuente: adaptado de [17]

Si bien es cierto, la prueba TRAM permite evaluar la calidad higiénica de la leche en campo [16], se hace necesario para futuros estudios hacer un conteo más específico sobre los microorganismos presentes en este tipo de leches. Por ejemplo, existen varios factores que pueden afectar el tiempo de reducción en la prueba, algunos de ellos son el tipo de microorganismos, el número de leucocitos, el periodo de exposición a la luz, la cantidad de oxígeno disuelto [30], estos toman importancia sobre la necesidad de evaluar específicamente el tipo de microorganismos presentes, pues cada grupo interfiere en procesos distintos al igual que genera impactos sobre la calidad higiénica en diferente grado [30]. 
Por otro lado, mediante la prueba de CMT se encontró durante el primer periodo de muestreos que el $79,31 \%$ de los medios evaluados en las UP resultaron negativos, el 8,97\% presentó mastitis subclínica tipo 1, mientras que el 6,21 \% y el 5,52 \% fue diagnosticado con mastitis tipo 2 y tipo 3 , respectivamente. Para el segundo periodo de tiempo, el 75,61 \% resultaron negativos, el 9,35\% presentó mastitis subclínica tipo 1, el 8,94 \% tipo 2, el 5,28 \% tipo 3, solo durante este periodo, el 0,81 \% presentó casos positivos o clínicos de mastitis, lo que evidenció que para el segundo periodo se incrementan los casos subclínicos de mastitis, pasando de un 20,69 \% en el primer periodo a un 23,57 \% en el periodo 2, con un único caso de mastitis clínica (ver tabla 4), estos resultados alertan sobre posibles acciones de control sanitario y de manejo que se deban aplicar, además de iniciar con los procesos de certificación en buenas prácticas de ordeño de acuerdo con la normatividad.

Tabla 4. Prevalencia de mastitis clínica y subclínica en las muestras evaluadas en las UP en dos periodos de tiempo

\begin{tabular}{lccccc}
\hline \multirow{2}{*}{ CMT } & CS/ml & \multicolumn{2}{c}{ Periodo 1 } & \multicolumn{2}{c}{ Periodo 2 } \\
\cline { 3 - 6 } & & $\mathbf{n}$ & $\mathbf{\%}$ & $\mathbf{n}$ & \% \\
\hline Negativo & $<500.000$ & 230 & 79,31 & 186 & 75,61 \\
\hline Subclínica 1 & $500.000-900.000$ & 26 & 8,96 & 23 & 9,35 \\
\hline Subclínica 2 & $900.001-5{ }^{\prime} 000.000$ & 18 & 6,21 & 22 & 8,94 \\
\hline Subclínica 3 & $>5^{\prime} 000.001$ & 16 & 5,52 & 13 & 5,28 \\
\hline Positivo (Clínica) & & 0 & 0,00 & 2 & 0,81 \\
\hline Total medios evaluados & & $\mathbf{2 9 0}$ & $\mathbf{2 4 6}$ & \\
\hline Total animales & $\mathbf{1 4 5}$ & $\mathbf{1 2 3}$ & \\
\hline
\end{tabular}

CMT: California Mastitis Test, CS: células somáticas

Fuente: elaboración propia.

Este incremento en la presencia de mastitis durante el periodo 2 implica un deterioro en la calidad higiénica de la leche, además, se ha relacionado que la presencia de animales con mastitis puede provocar un incremento en las UFC, teniendo en cuenta que, una leche con mastitis clínica presenta entre 6 y 7 millones de UFC/ml [17]. En los resultados puede observarse que el CMT está relacionado con la presencia de leches grado I, lo que indica que se deben valorar las condiciones de los protocolos de manejo en la rutina de ordeño, los cuales pueden estar repercutiendo directamente en la salud de la glándula mamaria, así como, los procesos de manejo de la leche en su almacenamiento y transporte están afectando la calidad higiénica. 
A partir de estos resultados que muestran el comportamiento de las variables fisicoquímicas: grasa, proteína, SNG, densidad, punto crioscópico y de la prueba CMT para determinar la presencia de células somáticas en glándula mamaria y posible presencia de microorganismos contaminantes- UFC a partir de la prueba indirecta TRAM, se cuenta con valores de referencia que pueden servir como línea base de información para continuar explorando los diferentes factores que afectan la calidad total de la leche y orientar la estructuración de aspectos normativos para la regulación de los procesos de comercialización y condiciones de inocuidad para el consumo humano, en concordancia con la exigencia que se tiene para la leche de vaca, reglamentada en el Decreto 616 de 2006.

\section{Conclusiones y recomendaciones}

Se encontró que en la leche obtenida en las UP de la provincia de García Rovira los niveles de grasa, proteína, sólidos no grasos, densidad y punto crioscópico se encuentran dentro del rango de los valores reportados para la leche de cabra en diferentes lugares del mundo. Según la prueba de California Mastitis Test, cerca del 80 \% de los animales evaluados son negativos a la presencia de células somáticas en la leche y según la prueba TRAM, el 73 \% de la leche se califica como grado II y III, la cual es aceptada para el consumo humano. Estos datos contribuyen con información referencial fundamental que permiten apoyar la estructuración de aspectos de carácter normativo para el consumo de leche de cabra en el país; es preciso trabajar en recopilar más información procedente de sistemas de producción con una adecuada trazabilidad, que considere los diferentes factores genéticos, nutricionales, sanitarios, de manejo y ambientales que afectan las características de calidad de la leche.

\section{Agradecimientos}

A Lácteos Rovirenses S. A. Por su apoyo en el desarrollo del proyecto enmarcado en la ejecución de esta investigación.

\section{Referencias}

[1] Atto-Mendives JA. Importancia de los ovinos tropicales introducidos al pais: Características Productivas y Reproductivas. Arch Latinoam Prod Anim. 2007;15(1):310-5. 
12 Características composicionales y calidad higiénica de leche de cabra de sistemas de producción de la provincia de García Rovira, Santander (Colombia)

[2] De Vries J. Goats for the poor: Some keys to successful promotion of goat production among the poor. Small Rumin Res. 2008;77(2-3):221-4. doi: https://doi.org/10.1016/j. smallrumres.2008.03.006

[3] Organization Food and Agriculture. FAOSTAT. 2017. [Internet]. [Citado en 3 de julio de 2018]: http://www.fao.org/faostat/en/\#compare;

[4] Instituto Colombiano Agropecuario. Inventario Caprino Municipios y Departamentos. 2020. [Internet]. [Citado en 3 de julio de 2020]: ://www.ica.gov.co/areas/pecuaria/servicios/ epidemiologia-veterinaria/censos-2016/censo-2018

[5] Cadena productiva Ovino Caprina Nacional. Acuerdo Nacional de Competitividad. Ministerio de Agricultura. 2012. 89 p.

[6] Vargas-Bayona JE, Serrano-Novoa C, Martínez-Bello D, Rodríguez-Galván A, MartínezZaragoza L, Serrano-Mujica LK. Los sistemas de producción caprina en el municipio de Molagavita, Santander sobre la cuenca del río Chicamocha. Spei Domus. 2015;11(23):8-16. doi: https://doi.org/10.16925/sp.v11i23.1362

[7] Moreno DC, Grajales HA. Caracterización de los sistemas de producción ovinos de trópico alto en Colombia: manejo e indicadores productivos y reproductivos. Rev la Fac Med Vet y Zootec. 2017;64(3):36-51. doi: https://doi.org/10.15446/rfmvz.v64n3.68693

[8] Koluman Darcan N, Silanikove N. The advantages of goats for future adaptation to Climate Change: A conceptual overview. Small Rumin Res. 2018;163:34-8. doi: https://doi.org/10.1016/j.smallrumres.2017.04.013

[9] Tarola AM, Preti R, Girelli AM, Campana P. Recent Advances in the Valorization of Goat Milk: Nutritional Properties and Production Sustainability. Nutr Food Eng. 2019;13(6):183-8. doi: doi.org/10.5281/zenodo.3299563

[10] Sepe L, Argüello A. Recent advances in dairy goat products. Asian-Australasian J Anim Sci. 2019;32(8):1306-20. doi: 10.5713/ajas.19.0487

[11] Yangilar F. As a Potentially Functional Food: Goats' Milk and Products. J Food Nutr Res. 2013;1(4):68-81. doi: 10.12691/jfnr-1-4-6

[12] Arechiga C, Aguilera JI, Rincón R, Méndez de Lara S, Bañuelos V, Meza-Herrera C. Situación actual y perspectivas de la producción caprina ante el reto de la globalización. Trop Subtrop Agroecosystems. 2008;9(1):1-14. 
[13] Bolacali M, KÜÇÜK M. Fertility and Milk Production Characteristics of Saanen Goats Raised in Muş Region. Kafkas Univ Vet Fak Derg. 2012;18(3):351-8.

[14] Guzmán G. The Chicamocha river canyon. En: Hermelin M, editor. Landscapes and Landforms of Colombia. 1st ed. Springer International Publishing; 2015. p. 73-83.

[15] García ME, Fuentes LA, Fernández SI. Determinación de la calidad higiénica de la leche mediante la medición indirecta del tiempo de reducción del azul de metileno o prueba de la reductasa microbiana. ETSIAMN Univ Politècnica València [Internet]. 2014 [citado 22 de 2018]. Disponible en: https://riunet.upv.es/bitstream/handle/10251/38380/Eva García. Calidad leche-2014.pdf?sequence=1

[16] Zambrano J, Grass-Ramírez J. Valoración de la calidad higiénica de la leche cruda en la asociación de productores de leche de Sotará-ASPROLESO, mediante las pruebas indirectas de resazurina y azul de metileno. Fac Ciencias Agropecu. 2008;6(2):56-66.

[17] Morales-Vanegas S de los A. Predicción de conteos microbiológicos en la leche cruda con base en la prueba de azul de metileno. [Tesis]. Honduras: Universidad Zamorano; 2005.

[18] Radostits OM, Done SH, editors. Veterinary medicine: a textbook of the diseases of cattle, sheep, pigs, goats, and horses. 10ª ed. New York: Elsevier Saunders; 2007.

[19] Faria Reyes FF, García A, Allara M, García A, Olivares Y, Ríos G. Algunas características fisíco-químicas y microbiológicas de la leche de cabra producida en Quisiro. Rev Fac Agron. 1999;16:99-106.

[20] Bedoya MO, Posada SL, Rosero NR. Composición de la leche de cabra y factores nutricionales que afectan el contenido de sus componentes. Corporación Universitaria Lasallista. 2012.

[21] Chacón-Villalobos A. Aspectos Nutricionales de la leche de cabra (Capra hircus) y sus variaciones en el proceso agroindustrial. Agron Mesoam. 2005;16(2):239-52.

[22] Gómez-Cortés P, Juárez M, De la Fuente M. Milk fatty acids and potential health benefits: An updated vision. Trends Food Sci Technol. 2018;81(agosto):1-9. doi: 10.1016/j.tifs.2018.08.014

[23] Ocampo R, Gómez A, Restrepo D, Cardona H. Estudio comparativo de parámetros composicionales y nutricionales en leche de vaca, cabra y búfala, Antioquia, Colombia. Rev Colomb Cienc Anim. 2016;8(2):177-86. doi: https://doi.org/10.24188/recia.v8.n2.2016.185 
14 Características composicionales y calidad higiénica de leche de cabra de sistemas de producción de la provincia de García Rovira, Santander (Colombia)

[24] Herrera-Campos L, Vargas-Rodríguez C, Boschini-Figueroa A, Chacón-Villalobos A. Variación bromatológica de la leche de cabras Lamancha alimentadas con diferentes forrajes. Agron Mesoam. 2009;20(2):381-90. doi: 10.15517/am.v20i2.4954

[25] Kljajevic NV, Tomasevic IB, Miloradovic ZN, Nedeljkovic A, Miocinovic J, Jovanovic S. Seasonal variations of Saanen goat milk composition and the impact of climatic conditions. J Food Sci Technol. 2017;55(octubre):299-303.

[26] Ibrahim Sabah IO, Awadelkareem AM, AshrafSA, Sabahelkhier MK. Comparative studies on the physicochemical and microbiological charactristics of different animal milk collected from the farms of Khartoum State, Sudan. Biosci Biothechnology Res Commun. 2018;11(3):387-92.

[27] Elmaz O, Tasci F, Akbas A, Saatc M. First lactation milk yield, composition and some udder measurements of Honamh goats raised under extensive conditions. Anim Sci Pap Reports. 2018;36(4):393-403.

[28] Sanz CL, Ramos M, Gloria de la Torre A, Diaz C, Pérez M, Sanz S. Composition of goat and cow milk produced under similar conditions and analyzed by identical methodology. Journal Food Compos Anal. 2009;22(4):322-9.

[29] Milewski S, Katarzyna Z, Antoszkiewicz Z, Tański Z, Sobczak A. Impact of production season on the chemical composition and health properties of goat milk and rennet cheese. Emirates J Food Agric. 2018;30(2):107-14.

[30] Universidad de Zulia. Introducción al control de calidad de la leche cruda. 2003. (1). Report No.: 1. 